\title{
Tumor Location and Nature of Lymphatic Vessels are Key Determinants of Cancer Metastasis
}

\author{
Ramin Shayan * $,+\star, \S$, Rachael Inder *, Tara Karnezis *, , Carol Caesar ${ }^{*, \S,}$ \\ Karri Paavonen *, Mark W. Ashton ${ }^{\star}$, G. Bruce Mann ${ }^{\dagger}$, G. Ian Taylor ${ }^{\star}$, \\ Marc G. Achen ${ }^{*} \S$ and Steven A. Stacker *,§
}

\begin{abstract}
*Ludwig Institute for Cancer Research, Post Office Box 2008 Royal Melbourne Hospital, Victoria 3050, Australia

${ }^{\dagger}$ Department of Surgery, Royal Melbourne Hospital, University of Melbourne, Victoria 3050, Australia

†Jack Brockhoff Reconstructive Plastic Surgery Research Unit, Royal Melbourne Hospital and Department of Anatomy, Faculty of Medicine, Dentistry and Health Sciences, University of Melbourne, Victoria 3050, Australia.

${ }^{\S}$ New Address: Tumour Angiogenesis Program, Peter MacCallum Cancer Centre, St. Andrews Place, 3002, Melbourne, Australia and Sir Peter MacCallum Department of Oncology
\end{abstract}

Running Title: Tumor location and cancer metastasis

Financial Support: This work was funded partly by a Program Grant from the National Health and Medical Research Council of Australia (NH\&MRC). SAS and MGA are supported by Senior Research Fellowships from the NH\&MRC. SAS would like to 
acknowledge the support of the Pfizer Australia Fellowship. RS is supported by the Raelene Boyle Sporting Chance Foundation and Royal Australasian College of Surgeons (RACS) Foundation Scholarship, and the RACS Surgeon Scientist Program. This work was supported by funds from the Operational Infrastructure Support Program provided by the Victorian Government, Australia.

Correspondence and requests for materials should be addressed to:

Steven A. Stacker, Peter MacCallum Cancer Centre, St Andrews Place, East Melbourne, Victoria 3002, Australia. Email:Steven.stacker@ petermac.org. Phone: +613-9656-5263

Fax: +613-9656-1411

SAS and MGA are consultants to Vegenics Ltd and are stock holders in Circadian Technologies. 


\section{ABSTRACT}

Tumor metastasis to lymph nodes is a key indicator of patient survival, and is enhanced by the neo-lymphatics induced by tumor-secreted VEGF-C or VEGF-D, acting via VEGFR-3 signalling. These targets constitute important avenues for anti-metastatic treatment. Despite this new understanding, clinical observations linking metastasis with tumor depth or location suggest that lymphangiogenic growth factors are not the sole determinants of metastasis. Here we explored the influence of tumor proximity to lymphatics capable of responding to growth factors on nodal metastasis in a murine VEGF-D over-expression tumor model. We found that primary tumor location profoundly influenced VEGF-D-mediated lymph node metastasis: $89 \%$ of tumors associated with the flank skin metastasised, in contrast with only $19 \%$ of tumors located more deeply on the body-wall $(\mathrm{p}<0.01)$. Lymphatics in metastatic tumors arose from small lymphatics, and displayed distinct molecular and morphological profiles compared with those found in normal lymphatics. Smaller lymphatic subtypes were more abundant in skin $(2.5$-fold, $\mathrm{p}<0.01)$ than in body wall, providing a richer source of lymphatics for VEGF-D $^{+}$skin tumors, a phenomenon also confirmed in human samples. This study shows that the proximity of a VEGF- ${ }^{+}$primary tumor to small lymphatics is an important determinant of metastasis. These observations may explain why tumor location relative to the lymphatic network is prognostically important for some human cancers.

Key Words: Metastasis •lymph node $\bullet$ lymphatics $\bullet$ prognosis $\bullet$ VEGF-D 

Abbreviations :
BW Body Wall
DAB Diaminobenzidene
IL Initial Lymphatics
LEC Lymphatic Endothelial Cell
LN Lymph Node
LVD Lymphatic Vessel Density
LYVE-1 Lymphatic Vessel Endothelial Hyaluronan Receptor
$\mathrm{Np} \quad$ Neuropilin
PC Pre-Collector
PECAM-1 Platelet Endothelial Cell Adhesion Molecule-1
SCID/NOD Severe combined immunodeficiency/non-obese diabetic mice
SK Skin
SMC Smooth Muscle Cell
VEGF Vascular Endothelial Growth Factor 


\section{INTRODUCTION}

Lymph node (LN) involvement is an important diagnostic and prognostic parameter for the clinical management of many human tumors [1, 2]. Clinical and experimental data indicate that LN metastasis is associated with tumor-related lymphangiogenesis (lymphatic vessel growth), which is promoted by the expression of the lymphangiogenic growth factors VEGF-C or VEGF-D[3-5], or VEGF-A [6] in mouse cancer models . These findings are supported by histopathological studies of human tumors in which VEGF-C or VEGF-D expression correlated with increased lymphatic vessel density (LVD), in the primary tumor, and with metastasis [7-9]. Consequently, molecules such as VEGF-C or VEGF-D, and their signalling pathway via the VEGFR3/Npr-2 receptor complex, are important targets for anti-lymphangiogenic treatment as an avenue to inhibit tumor metastasis $[3,5,10,11]$, for review see [12].

Apart from 'LN-status', parameters such as tumor depth/location, mitotic-rate and nuclear/cytoplasmic ratio are also useful prognostic indicators that guide clinical management of cancer patients, depending on the tumor type[13]. Immunohistochemical correlation between lymphangiogenic growth factor expression and nodal spread in patients has led to suggestions that VEGF-C and VEGF-D may be independent markers of metastatic risk and poor prognosis [14-16] (summarised in [13, 17-20]). Nevertheless, the size and location of a tumor, and depth of tumor invasion remain key indicators in the pathological assessment of many types of cancer. For example, the depth of an invading melanoma 'cell front' is a key prognostic determinant; and more recently, it was observed that invasive breast cancers involving or near overlying skin have higher metastasis rates 
and worse prognoses than size-matched tumors located more deeply[21]. Further, the deepest tumor fronts in colorectal cancer express higher VEGF-C or VEGF-D levels than more superficial tumor tissue, and expression of these growth factors is independently related to poor prognosis[22]. In contrast, large, high-grade sarcomas rarely spread via lymphatics, despite sarcoma cells being able to interact with lymphatic endothelial cells (LECs) in vitro [23], but metastasise lymphogenously when grown near the skin[24]. This suggests that, in addition to requisite lymphangiogenic growth factors, other features such as the anatomical tumor location remain important determinants of LN spread[21]. However, the exact reason for this remains unclear.

It has been previously recognised that different subtypes of lymphatic vessels vary in morphology, anatomical location and embryological development; and perform distinct functional roles [25-30]. In skin, the lymphatic vasculature commences in the superficial dermis as 'initial' or 'capillary' lymphatics, which absorb lymph fluid. This fluid is then transported through deep dermal 'pre-collector' lymphatics, to the more deeply located 'collecting' lymphatics[25-29]. The latter have valves, a basement membrane, a smooth muscle cell (SMC) [31] and pericyte coating[27, 32], and a LEClined lumen that channels lymph to LN [25-29]. Currently, our ability to distinguish 'absorptive' from 'conductive' lymphatic vessels combines assessments of anatomical location, morphology, and lymphatic-marker profile [26-29, 32]. Given that distinct lymphatic subtypes have different distributions within the body, it is possible that the effect of primary tumor location on metastasis is based, in part, on the degree to which tumor-derived lymphangiogenic growth factors can access lymphatics with 
responsiveness to these ligands. The fact that the presence of SMCs and pericytes on certain blood vessels is thought to mediate a 'non-sprouting' or quiescent vessel phenotype [31] raises the possibility that different lymphatic subtypes also behave differently in response to lymphangiogenic growth factors [27, 32]. For example the specific response of collecting lymphatic vessels to VEGF-D involving prostaglandin signalling leading to vessel dilation to favour transport of tumor cells, has recently been established [33].

Here we explore the significance of tumor location for VEGF-D-induced tumor lymphangiogenesis, and examine how the nature of the nearby lymphatic vessels influences the generation of 'absorptive' tumor lymphatics[34], and thereby, tumor metastasis. We also define tumor lymphatics in terms of features that distinguish normal lymphatic subtypes. Our findings suggest that initial/capillary lymphatics and precollecting lymphatics (referred to collectively as small lymphatics) are the source vessels that sprout to form tumor 'neo-lymphatics'; and that VEGF-D-expression in mouse tumors induces sprouting from these smaller calibre vessels but not larger collecting lymphatics. While VEGF-D expression does enhance LN metastasis, this process relies on the presence of lymphatics that are able to sprout in response to the lymphangiogenic growth factors. This effect is verified using equivalent VEGF-C over-expressing tumors. Therefore, variations in the lymphatic subtypes occuring in different locations of the body may be an important determinant of tumor metastasis. 


\section{MATERIALS AND METHODS}

\section{Antibodies}

Primary antibodies used: MAb against mouse PECAM-1 (1:1000) (BD Pharmingen, San Diego, CA), collagen type IV (1:300) and M2 (anti-FLAG) (1:500) (Sigma, St. Louis, MO); LYVE-1 (1:1000) and podoplanin (1:200) (R\&D Systems, Minneapolis, MN); human LYVE-1 (1:1000) and VEGF-D (1:50) (R\&D Systems, Minneapolis, MN), SMA (1:500) (DAKO, Carpinteria, CA) and human D2-40 antibody to podoplanin (1:500) (Vector Laboratories, Burlingame, CA). Polyclonal antibodies used: biotin-conjugated anti-rat Ig (DAKO Corp., Carpinteria, CA), peroxidaseconjugated Ig against rat (DAKO Corp., Carpinteria, CA), mouse (Bio-Rad, Hercules, CA), rabbit and goat Ig (1:1000) (DAKO) and rabbit anti-mouse LYVE-1 (1:1000) (Fitzgerald Industries, Concord, MA) and NG2 antibody (1:200) to pericytes (Milipore, Billerica, MA). Phalloidin (Sigma, St. Louis, MO) was used to detect F-actin.

Alexa fluorescent-conjugated secondary antibodies included anti-rat, anti-rabbit, anti-mouse and anti-hamster antibodies (used at 1:500) (Invitrogen, Carlsbad, CA). Immunostaining of paraffin and frozen sections involved a $\mathrm{DAB}$ chromogen, with $\mathrm{ABC}$ amplification (Vector Laboratories, Burlingame, CA) when required, or fluorescent samples were mounted using SlowFade Antifade kit, (Invitrogen, (Molecular Probes), Carlsbad, CA), as per manufacturer's instructions.

\section{Generation and analysis of tumors and wound samples}

293EBNA-1 flank [5] and ear tumors were established as described[35-37], in SCID/NOD mice (ARC, Perth, Australia) and animals sacrificed prior to tumors reaching 
a volume of $2500 \mathrm{~mm}^{3}$ (flank) or a diameter of $2 \mathrm{~mm}$ (ear) (as per local Animal Ethics Committee limits). Inhalational anaesthesia (4\% isoflurane in $3.0 \mathrm{~L} / \mathrm{min}$ oxygen administered for 1 minute, then adjusted to $3 \%$ isoflurane in $0.2 \mathrm{~L} / \mathrm{min}$ oxygen for maintenance) administered via a TM41 CIG anaesthetic machine (North Ryde, New South Wales, Australia) was used to perform tumor inoculations, and animals were euthanized using inhaled carbon monoxide (as per local Animal Ethics Committee specifications). Tumors and regional LNs were removed post mortem for histological examination. Three other constructs were made in which either or both of the N- or Cterminal domains of human VEGF-D were deleted; VEGF-D $\Delta \mathrm{N} \Delta \mathrm{C}[38]$, VEGF-D $\Delta \mathrm{C}$ and VEGF-D $\Delta$ N[39]. VEGF-D $\Delta$ N consists of residues 93 to 354 of human VEGF-D tagged at the N-terminus with the FLAG octapeptide. The three amino acid residues $\mathrm{T}, \mathrm{R}$ and $\mathrm{N}$ are present between FLAG and the VEGF-D sequence. This protein lacks the N-terminal propeptide of VEGF-D. Each of these constructs contained a FLAG sequence to facilitate protein purification. Cell lines expressing a combination of mouse VEGF- $\mathrm{A}_{164}$ and human VEGF-C were also generated in 293-EBNA-1 cells and formed the basis of control tumors.

Eighty-four VEGF-D ${ }^{+}$and twenty VEGF-D ${ }^{-}$flank tumors were retrospectively grouped as being associated with the body wall (BW) or with the overlying skin (SK), and LN were analyzed for metastatic tumor cells. Tumor and normal tissue sections were immunostained for LYVE-1, podoplanin, aSMA, F-Actin, type IV collagen and NG2. Mouse tissues were harvested and either frozen in Tissue-Tek OCT (Sakura, Tokyo, Japan) or fixed in $4 \%$ paraformaldehyde (PFA) prior to paraffin embedding or wholemount staining. Wholemount ear samples were immunofluorescently stained using 
overnight incubations $\left(4^{\circ} \mathrm{C}\right.$ with gentle agitation) for LYVE-1 or podoplanin as previously described [29]. Samples were analysed using a fluorescence Nikon Eclipse 90i upright microscope with a Nikon DXM1200c camera, and Nikon TE2000-E and C1 confocal microscope. Microwave antigen retrieval of paraffin embedded sections was performed with $\mathrm{pH} 6.0$ citrate buffer, at approximately $90^{\circ} \mathrm{C}$ to $94^{\circ} \mathrm{C}$ for two consecutive treatments of five minutes each (citrate buffer was topped-up between), at high setting. The left ears of 20 female SCID/NOD and 15 C57Bl/6 mice (WEHI, Melbourne, Australia) were wounded with a standard punch [40] and harvested at day 21.

\section{ELISA, Western blotting and immunoprecipitation studies.}

ELISA for VEGF-D was performed using an R\&D Systems kit (Minneapolis, $\mathrm{MN})$ as per manufacturer's instructions, and tumor samples tested for VEGF-D expression by immunoprecipitation followed by Western blot as described previously [5, $38]$.

\section{Human tissue samples}

Human tissue samples were surplus tissue from reduction mamoplasty surgery, that was collected at the time of surgery with appropriate institutional approval and patient consent. Tissues were fixed in $4 \%$ PFA prior to paraffin embedding and immunohistochemical vessel quantification analysis (see below). 


\section{Lymphatic quantification and statistical analysis}

Quantification of LVD within flank tumors was performed using a pixel counting method in which multiple areas of LYVE-1-stained tumor sections were photographed and the identified vessels were quantified using Corel Draw software (Coral Draw Graphics Suite X3, Corel Corporation, Ottawa, Canada), as previously described [5]. Lymphatic vessels in normal ear skin, or wounded ear skin or ear tumor models were quantified following immunofluorescent wholemount staining with LYVE-1 or podoplanin, using the LVAP software plugin for ImageJ [37]. Similarly, skin and body wall lymphatics in both animal and human specimens were quantified using the LVAP software on immunohistochemical sections, once the lymphatic vessels had been identified using a combination of anti-LYVE and anti-podolplanin vessel staining techniques.

Statistical analyses were performed as previously described [5], using two-tailed student's T-test. Statistical significance was attributed to $\mathrm{p}$ values $<0.05$. 


\section{RESULTS}

\section{Primary tumor location influences metastasis to regional LNs.}

We previously showed that tumor xenografts generated from 293EBNA-1 transformed embryonal kidney cells do not metastasize; however, when stably transfected to express VEGF-D, they frequently do so [5]. This model was found to have no baseline level of VEGF-C or VEGF-A secretion [5]. In order to assess the effect of primary tumor location on LN metastasis, we established 293EBNA-1 tumors expressing full-length human VEGF-D (VEGF-D ${ }^{+}$tumors) and control tumors, expressing no VEGF-D (VEGF$\mathrm{D}^{-}$tumors), in the flanks of SCID/NOD mice [5]. When the flank skin was reflected to reveal the primary tumors post-mortem, they could be classified as either adherent to skin (designated "SK" tumors) or to the underlying musculature of the body wall (designated "BW" tumors) (Fig. 1A). Histological examination of ipsilateral axillary LNs revealed that SK tumors spread to LNs much more frequently than BW tumors (40/45 or 89\% SK tumours spread to LNs; $3 / 16$ or $19 \%$ BW tumors spread to LNs; p value < 0.01) (Fig. 1B). In contrast, none of the VEGF-D ${ }^{-}$tumors, be they SK or BW tumors, metastasised (Fig. 1B). The sizes of SK and BW VEGF-D ${ }^{+}$tumors were not significantly different (both were $600-700 \mathrm{~mm}^{3}$ after 23 days and grew at similar rates throughout), and comparable VEGF-D-expression was detected in both tumor types as assessed by immunoblotting and ELISA (Fig. 1B). In order to exclude these findings being specific to this lymphangiogenic growth factor over-expression model, similar studies using variant forms of VEGF-D and VEGF-C were performed. Similar results were observed with 293EBNA-1 tumors secreting variant forms of the VEGF-D polypeptide (VEGF-D $\Delta \mathrm{N} \Delta \mathrm{C}$, SK 13/16 metastasis to regional lymph node, BW 0/8; VEGF-D $\Delta$ C, SK6/10, BW 2/8; 
VEGF-D $\Delta$ N, SK 14/17, BW 1/10) or with tumors over-expressing a combination of other lymphangiogenic growth factors, mouse VEGF-A164 and VEGF-C (SK 12/14, BW 0/4) (data not shown)

To explore the discrepancy found between the rates of LN metastasis of SK and BW tumors - and the role played by the differences in lymphatic subtypes in the two anatomical locations - immunohistochemistry and quantitation of tumor lymphatics were performed (Fig. 1C and D) using antibodies to podoplanin [27] and LYVE-1 [4, 5], LECexpressed proteins commonly used to analyse lymphatics. The use of multiple LEC markers showed that most tumor lymphatics co-expressed LYVE-1 and podoplanin (Supplemental Fig. 1). Tumor LVD quantitation using LYVE-1, as shown in Fig. 1D, demonstrated that SK tumors had a 3.5-fold higher LVD than BW tumors ( $\mathrm{p}<0.01)$ (Fig. 1C). In addition, tumor lymphatics in SK tumors were morphologically abnormal (Fig. 1D upper and lower left panels). In general, LYVE- $1^{+} /$podoplanin $^{+}$lymphatics in metastatic VEGF-D ${ }^{+}$SK tumors were predominantly peripherally located, extending for variable distances towards the tumor centre, often spanning up to half the tumor tissue sections (approximately $300 \mu \mathrm{m}$ ). On many occasions, individual vessels were continuous in multiple serial sections for up to $200 \mu \mathrm{m}$. In contrast, the few LYVE$1^{+}$podoplanin $^{+}$lymphatics present in indolent VEGF-D ${ }^{+}$BW and VEGF-D $^{-}$control tumors, were not highly branched or dilated and were not seen in multiple serial sections (Fig. 1D upper right panel). Staining with an antibody to smooth muscle actin (SMA) to ascertain whether SMCs were present on tumor lymphatics $[29,41]$ revealed a variable and patchy covering of $\mathrm{SMA}^{+}$cells on the $\mathrm{LYVE}-1^{+} /$podoplanin $^{+}$tumor lymphatic 
population (Fig. 1D lower right panel and Supplemental Fig. 2). In addition, staining for F-Actin, collagen IV and the NG2 antibody revealed an absence of anchoring filaments and pericytes, and scant basement membrane on tumor lymphatics (data not shown).

\section{Small lymphatic subtypes are more abundant in the skin than the body wall.}

The recent description of lymphatic vessel subtypes in terms of different LEC marker profiles has improved immunohistochemical differentiation of these subtypes in tissue samples [25-29]. While dermal initial lymphatics were shown to express both LYVE-1 and podoplanin, LECs in the subcutaneous collecting lymphatics express podoplanin, but not LYVE-1, and are associated with mural SMCs [25-29]. Therefore, to identify the lymphatic vessels in mouse skin and body wall, mouse flank tissue sections were stained for LYVE-1 and podoplanin (Fig. 2A).

LYVE- $1^{+} /$podoplanin $^{+}$structures (Fig. 2A), consistent with initial and precollector lymphatics (so-called 'small' lymphatics) [25-29], were located in both the body wall and skin (Fig. 2A upper panels) of the mouse flank. We did not differentiate between initial and pre-collector lymphatics due to similar morphology, staining profiles and anatomical location within these regions. An additional subset of LYVE-1' /Podoplanin ${ }^{+}$lymphatic vessels (Fig. 2A lower panels) present in both body wall and skin is consistent with the expected staining profile of collecting lymphatics [25-29]. The level of LYVE-1 staining on these vessels was consistent with that of negative control antibodies (data not shown) 
Using this differential expression profile of LEC-markers to identify the respective lymphatic vessel subtypes, quantitation of the density of LYVE$1^{+} /$Podoplanin $^{+}$('small' lymphatics) vs. LYVE-1/20doplanin ${ }^{+}$(collecting lymphatics) within the skin and body wall was performed (Fig. 2B). Serial mouse flank sections revealed that, although a similar density of collecting lymphatics were present in the body wall as in skin; there was a significant difference between the densities of the other lymphatic subtypes present in the two locations (Fig. 2B). The average density of 'small' lymphatics (a combination of initial and pre-collecting vessels) was $\sim 2.5$-fold higher in skin than that in the body wall (Fig. 2B).

In order to establish if the difference in the density of small lymphatic vessels observed between mouse skin and body wall applies in human tissues, samples extending continuously from skin to deep fascia (inclusive) were taken from six breast reduction operations (Fig. 2C left panel) and stained for podoplanin (using anti-human D2-40) and LYVE-1 (Fig. 2C right panel). Quantitation of the density of LYVE-1 $1^{+} / \mathrm{D} 2-40^{+}$small lymphatics (Fig. 2D and Supplemental Fig. 3) and LYVE-1 $1 / \mathrm{D} 2-40^{+}$collecting lymphatics (Fig. 2D and Supplemental Fig. 4) in these human tissues demonstrated a similar ratio between the lymphatic subtypes in the skin and those in deeper tissues, as seen in the same comparison of mouse tissue (Fig. 2B and 2D), i.e. whilst there is a similar density of collecting lymphatics in both the skin and body wall, there is a significantly greater density of 'small' lymphatics in the skin compared with that in the body wall (Fig. 2B and 2D). 


\section{Ear tumor models identify the presence and origin of pathological lymphatics.}

In order to explore the differences between lymphangiogenic responses of the distinct subtypes of lymphatic vessels and to enable us to examine lymphatics in metastatic VEGF-D ${ }^{+}$tumors using wholemount immunofluorescence, the VEGF- ${ }^{+}$flank tumor model was adapted to the ear as previously described (see methods) (Fig. 3A). None of the mice with VEGF-D ${ }^{-}$ear tumors had LN metastases to the regional draining LNs, the cervical LN chain; however, 14/15 mice with VEGF-D ${ }^{+}$ear tumors did so. Further, the morphological and molecular features of the lymphatics identified on immunostaining of metastatic ear tumor sections (Fig. 3B and Supplemental Fig. 5) were consistent with those described in VEGF- $\mathrm{D}^{+}$flank tumors, i.e. predominantly peripherally located, highly branched, LYVE- $1^{+} /$podoplanin ${ }^{+}$lymphatics, punctuated by dilated LEClined spaces and often extending over multiple serial sections (see Fig. 1D). Control VEGF-D ${ }^{-}$tumors lacked lymphatic structures (data not shown). In addition, as has been shown in VEGF-C over-expressing tumors [35, 42, 43], superficial dermal lymphatics adjacent to VEGF- $\mathrm{D}^{+}$tumors were dilated compared to lymphatics in the skin of normal control animals (Fig. 3A). Unlike the deep dermal lymphatics, the most superficial LYVE- $1^{+} /$podoplanin $^{+}$dermal lymphatics did not appear to be continuous with the large, deranged, irregular LYVE-1 $1^{+} /$podoplanin ${ }^{+}$lymphatics adjacent to the tumor mass (Fig. 3B).

To analyse sprouting from lymphatics in response to VEGF-D, wholemount immunofluorescence of ear tissue was first performed to verify our ability to differentiate normal lymphatic subtypes. A combined analysis of anatomical location, morphological 
characteristics and LYVE-1/podoplanin staining was used. Blind-ended, highly branched, strongly LYVE- $1^{+} /$podoplanin ${ }^{+}$initial lymphatics were seen, located more superficially to straighter, less branched, valve-bearing LYVE-1\%/podoplanin ${ }^{+}$collecting vessels (Fig. 3B) $[43]$.

In samples of ear skin bearing VEGF-D ${ }^{+}$tumors, deranged lymphatic structures were seen, that correlated with molecular and morphological features (Fig. B) observed in sections from metastatic VEGF-D ${ }^{+}$flank (Fig. 1D) or ear tumors (Fig. 3A), discussed above. VEGF-D ${ }^{+}$ear tumors induced small lymphatic vessel sprouts and connections were observed between tumor lymphatics and the native small lymphatic network (Fig. 3B). In contrast, neither lymphatic sprouting, nor any direct connections from tumor lymphatics were seen to involve the collecting lymphatics in the ear subcutis (Fig. 3B). Quantification of the number and characteristics of the sprouts arising in an ear tumor was performed and compared with those arising in the ear skin wounding model (Fig. 3C, discussed below).

\section{An ear wounding model verifies the nature of pathological lymphatic sprouting}

To analyse lymphangiogenesis in an alternative pathological setting, $2 \mathrm{~mm}$ circular ear wounds were created (Fig. 3D), as described [40]. Quantitative analysis of the resulting neo-lymphatics was performed (Fig. 3C) [37]. The newly-generated lymphatics

originated from pre-existing small lymphatics (as in the ear tumor model), and sprouted toward the wound stimulus (Fig. 3D, left panel). Quantification of the lymphatic sprouts driven by the two pathological models uncovered several differences. Compared with 
wound-induced lymphangiogenesis, ear skin influenced by VEGF-D ${ }^{+}$tumors displayed higher numbers of lymphatic sprouts, tips per sprout and lymphatic loops, however a shorter average sprout length was observed than in ear wounds (Fig. 3C).

\section{Lymphatic vessels in human tumors}

To confirm observations of the lymphatics in our animal models and to exclude the possibility that the characteristics of the mouse tumor lymphatics were attributable to xenograft transplantation alone[26], we investigated archived samples of human melanoma and surrounding skin, which were stained using the anti-human podoplanin [27] and anti-human LYVE-1 antibodies. Analysis revealed a similar abnormal lymphatic morphology (i.e. predominantly peripheral, disorganized, highly branched lymphatics) (Fig. 3D) as seen in our mouse tumor models. This pattern of staining was observed in 4 of 10 metastatic tumors but in none of 10 non-metastatic tumors analysed. 


\section{DISCUSSION}

Our results indicate that primary tumor location, particularly proximity to small lymphatic vessels, influences LVD in the tumor and LN metastasis. Two other possible explanations for the different rates of spread by SK and BW VEGF-D ${ }^{+}$tumors, that the tumors were of different sizes or exhibited different VEGF-D-expression, were excluded. On analysis of the primary tumors, the lymphatic structures seen in VEGF- ${ }^{+}$metastatic tumors exhibited morphology distinct from normal lymphatics. They underwent regular branching, occasional dilatation to form large LEC-lined spaces, and spanned multiple serial sections, suggesting expansive areas of LEC 'sheet-like' structures; all features reminiscent of those in VEGF-C over-expressing tumors [24, 42]. The lymphatics in the VEGF- $^{+}$metastatic tumors lacked basement membranes and pericytes, and were LYVE- $1^{+} /$podoplanin ${ }^{+}$, like normal lymphatic capillaries, but were deficient in anchoring filaments. The presence of SMC on tumor lymphatics is somewhat surprising, as LYVE1-expression has been shown to be down-regulated on LECs once SMCs become associated during lymphatic development [29] or regeneration [32]. Tumor lymphatics may, therefore, represent a distinct lymphatic subtype functionally adapted to favour LN metastasis. These tumor lymphatics displayed an enhanced surface area that facilitates interaction with tumor cells and growth factors, and encouraged further lymphatic sprouting to promote entry of metastatic cells to the lymphatic network. We have also previously observed altered gene expression of other growth factors in the endothelial cells of regional lymph nodes in this model associated with vascular remodelling and spread of tumor cells to regional lymph nodes[44]. 
It is likely that the difference between metastasis rates of SK and BW tumors was related to the different LVDs and/or altered lymphatic vessel morphology. Hence, the question arose as to why SK tumors were able to recruit neo-lymphatics more readily than BW tumors. The implication was that there may be differences in the character, type or density of the pre-existing lymphatics near the primary tumors, or in the ability of these vessels to respond to VEGF-D (for review see [45]). To investigate the lymphatic vessel subtypes in the normal flank, we differentiated between LYVE- $1^{+} /$podoplanin $^{+}$ 'small' (initial/capillary and pre-collector) and LYVE-1 ${ }^{1} /$ podoplanin ${ }^{+}$'large' (collecting) lymphatic vessels. Whilst there were similar densities of collecting lymphatics in the skin and body wall, there was a significant discrepancy between the densities of small lymphatics in these locations. Given that sprouting can occur from small lymphatics in response to VEGF-C [35, 36, 43] or VEGF-D [37], but has not been observed to occur from intact collecting lymphatics $[33,35,36,42,43]$, the difference in relative density of the lymphatic subtypes in the two locations could account for differences in the LVDs and thus metastasis rates of SK and BW tumors.

To test our hypothesis that small lymphatic subtypes were the active component of the skin that lead to the enhanced LVD in SK tumors, the flank tumor model was adapted to the ear, a location allowing visualisation and quantification of both normal and tumor lymphatics in 3-dimensions. VEGF-D-induced sprouts from nearby native small skin lymphatic vessels were continuous with tumor lymphatics, as were lymphatic sprouts driven by an alternative pathological stimulus, wound-healing. Together, these lymphatic sprouting models suggest that small-calibre lymphatics are the source of neolymphatics in pathological lymphangiogensis, in particular contributing to the enhanced 
LVD found in metastatic VEGF- ${ }^{+}$tumors. The high metastasis rate amongst VEGF-D ${ }^{+}$ ear tumors, despite smaller sizes than their flank counterparts, may occur because a tumor in the relatively thin ear will be nearer to skin; and the inability for VEGF-D ${ }^{-}$tumors to metastasise confirms the pre-requisite for a lymphangiogenic growth factor.

In contrast to small lymphatics, the collecting lymphatics in the ear did not sprout into or towards tumors. Larger blood vessels with associated mural SMC and pericytes are said to represent a 'quiescent, non-sprouting phenotype' [31, 46]. The low LVD in VEGF-D $^{+}$BW tumors that have access only to collecting lymphatics, and the lack of sprouting from collecting lymphatics near VEGF- ${ }^{+}$ear tumors, suggest that intact collecting lymphatics (which have a basement membrane, adventitia, and an SMC [32, 41] and pericyte coating [26, 28, 32]), may represent an analogous 'non-sprouting lymphatic phenotype'. Collecting lymphatics may therefore be unable to sprout towards tumors to form absorptive tumor lymphatics [34]. Intact collecting lymphatics may instead respond to lymphangiogenic growth factors by circumferential enlargement and dilatation $[35,36,42,43]$. This phenomenon was recently demonstrated in the case of VEGF-D mediated alterations, that were driven by enhanced levels of specific prostaglandins, and were related directly to rates of metastasis[33]. It is also consistent with previous observations of sprouting by small lymphatics, but only circumferential enlargement of collecting lymphatics, in models consisting of high-LVD metastatic VEGF-C over-expressing tumors [35, 36, 42, 43]. Collecting lymphatics, therefore, play an important, 'non-sprouting' role in LN metastasis [36] [33].

Overall, this study demonstrates that whilst lymphangiogenic growth factors are necessary for lymphatic vessel growth in tumors, they must have access to pre-existing 
lymphatics capable of responding to stimulatory cues. Our animal models have shown that primary tumor location exerts an effect on the ability to generate tumor lymphatics and thus promote metastasis, a hypothesis summarised in Fig. 4. Taken together, our data indicate that lymphatics grew into SK tumors from the skin in response to VEGF-D, establishing tumor lymphatics with a distinct morphological and molecular profile that promote metastasis. They indicate that while VEGF-D is permissive for lymphatic spread, its effect may be limited by the anatomical location of a tumor, and that access to lymphatic vessel subtypes capable of sprouting is critical for lymphangiogenic growth factors to generate tumor lymphangiogenesis and metastasis. In contrast, there is a paucity of small lymphatic vessels in the body wall that limits the abundance of lymphatics in BW tumors. Our results also indicate that human tumors formed near epithelial surfaces may have access, chiefly, to small lymphatics, rather than sparser, nonsprouting, collecting lymphatics to which more deeply-located tumors predominantly have access. Hence human tumors that may originate close to skin, such as breast carcinomas [21], would have a greater propensity to spread lymphogenously. Whilst VEGF-C or VEGF-D expression has been suggested as a prognostic indicator in several cancer types $[7,8,14,15]$, longstanding clinicopathological knowledge and more recent evidence that traditional indicators (such as the site of the tumor or tumor thickness and vertical growth phase in melanoma) remain key predictors of metastasis and determinants of clinical staging and prognosis $[1,21,47,48]$, should not be neglected. One of the limitations of this study is that other cell types in the tumor microenvironment that may additionally influence lymphangiogenesis have not been studied. Among these, macrophages have been suggested as key pro-lymphangiogenic cells during development, 
and lymphatic markers have been demonstrated on macrophage populations of the mouse embryo[49]. To counter this, however, the current tumor model was chosen due to low baseline levels of production of other lymphangiogenic growth factors in vitro and in vivo. In addition, the levels of these factors produced at a local level or secreted into the systemic circulation are no greater than baseline in the control tumor model, despite the presence of inflammatory cell populations. Further, recent studies have demonstrated that in the adult, inflammatory cells such as macrophages do not recapitulate their developmental role in lymphangiogenesis, as was once hypothesised[50].

We propose that the location of a primary epithelial tumor, when considered in conjunction with the level of VEGF-C or VEGF-D expression, derived from pathological samples, may provide early and accurate prognostic information regarding metastatic risk. The possibility that pathological tumor neo-lymphatics may express a novel molecular signature could provide additional avenues for identifying tumors that are more likely to spread, and may provide therapeutic targets for restricting metastasis[11]. 


\section{Acknowledgements}

The authors thank M. Kesar and staff at the Animal Facility at the Ludwig Institute for Cancer Research, Melbourne, for assistance with mouse experiments; M. Francois for provision of NG2 antibody; J. Taylor for assistance in generating figures; Y. Zhang for histology services; S. Cody for assistance with imaging; and A. Burgess and M. Hibbs for critical reading of this manuscript. Animal experiments and treatment of archived human tissue samples were in accordance with NH\&MRC guidelines. Ethics approval for research using human and animal samples was obtained from the Royal Melbourne Hospital Human Ethics Research Council, and the Ludwig Institute for Cancer Research Animal Ethics Committee, respectively. 


\section{REFERENCES}

1. Markovic SN, Erickson LA, Rao RD, et al (2007) Malignant melanoma in the 21st century, part 2: staging, prognosis, and treatment. Mayo Clin Proc 82: 490-513

2. DeVita VT, Hellman, S., Rosenberg, A. (2001) Cancer, principles and practice of oncology, 6th edn. Lippincott, Williams and Wilkins, Philadelphia, PA

3. Mandriota SJ, Jussila L, Jeltsch M, et al (2001) Vascular endothelial growth factor-C-mediated lymphangiogenesis promotes tumour metastasis. The EMBO journal 20: $672-82$

4. Skobe M, Hawighorst T, Jackson DG, et al (2001) Induction of tumor lymphangiogenesis by VEGF-C promotes breast cancer metastasis. Nature Med 7: 192-8

5. Stacker SA, Caesar C, Baldwin ME, et al (2001) VEGF-D promotes the metastatic spread of tumor cells via the lymphatics. Nature Med 7: 186-91

6. Nagy JA, Vasile E, Feng D, et al (2002) Vascular permeability factor/vascular endothelial growth factor induces lymphangiogenesis as well as angiogenesis. Journal of Experimental Medicine 196: 1497-506

7. Schietroma C, Cianfarani F, Lacal PM, et al (2003) Vascular endothelial growth factor-C expression correlates with lymph node localization of human melanoma metastases. Cancer 98: 789-97

8. White JD, Hewett PW, Kosuge D, et al (2002) Vascular endothelial growth factor-D expression is an independent prognostic marker for survival in colorectal carcinoma. Cancer Res 62: 1669-75 
9. Stacker SA, Williams RA, Achen MG (2004) Lymphangiogenic growth factors as markers of tumor metastasis APMIS 112: 539-49

10. Caunt M, Mak J, Liang WC, et al (2008) Blocking neuropilin-2 function inhibits tumor cell metastasis. Cancer Cell 13: 331-42

11. Achen MG, Mann GB, Stacker SA (2006) Targeting lymphangiogenesis to prevent tumour metastasis. BrJ Cancer 94: 1355-60

12. Achen MG, McColl BK, Stacker SA (2005) Focus on lymphangiogenesis in tumor metastasis. Cancer Cell 7: 121-7

13. Shayan R, Achen MG, Stacker SA (2006) Lymphatic vessels in cancer metastasis: bridging the gaps. Carcinogenesis 27: 1729-38

14. Goydos JS, Gorski DH (2003) Vascular endothelial growth factor C mRNA expression correlates with stage of progression in patients with melanoma. Clin Cancer Res 9: 5962-7

15. Nakamura Y, Yasuoka H, Tsujimoto M, et al (2005) Lymph vessel density correlates with nodal status, VEGF-C expression, and prognosis in breast cancer. Breast Cancer Res Treat 91: 125-32

16. Shayan R, Karnezis T, Murali R, et al (2011) Variations in tumor lymphatic density in primary cutaneous melanomas predicts risk of lymph node metastasis. Histopathology

17. Stacker SA, Achen MG, Jussila L, et al (2002) Lymphangiogenesis and cancer metastasis. NatRevCancer 2: 573-83

18. Alitalo K, Tammela T, Petrova TV (2005) Lymphangiogenesis in development and human disease. Nature (London) 438: 946-53 
19. Achen MG, Stacker SA (2008) Molecular control of lymphatic metastasis. Ann NY Acad Sci 1131: 225-34

20. Debinski W, Slagle-Webb B, Achen MG, et al (2001) VEGF-D is an Xlinked/AP-1 regulated putative onco-angiogen in human glioblastoma multiforme. Mol Med 7: 598-608

21. Cunningham JE, Juri AL, Oman L, et al (2006) Is risk of axillary lymph node metastasis associated with proximity of breast cancer to the skin? Breast Cancer Res Treat 100: $319-28$

22. Onogawa S, Kitadai Y, Tanaka S, et al (2004) Expression of VEGF-C and VEGF$\mathrm{D}$ at the invasive edge correlates with lymph node metastasis and prognosis of patients with colorectal carcinoma. Cancer Sci 95: 32-9

23. Bevacqua SJ, Welch DR, Diez de Pinos SM, et al (1990) Quantitation of human melanoma, carcinoma and sarcoma tumor cell adhesion to lymphatic endothelium. Lymphology 23: 4-14

24. Padera TP, Kadambi A, di Tomaso E, et al (2002) Lymphatic metastasis in the absence of functional intratumor lymphatics. Science 296: 1883-6

25. Smith KJ, Jones PF, Burke DA, et al (2011) Lymphatic vessel distribution in the mucosa and submucosa and potential implications for T1 colorectal tumors. Dis Colon Rectum 54: 35-40

26. Scavelli C, Weber E, Agliano M, et al (2004) Lymphatics at the crossroads of angiogenesis and lymphangiogenesis. J Anat 204: 433-49 
27. Van der Auwera I, Cao Y, Tille JC, et al (2006) First international consensus on the methodology of lymphangiogenesis quantification in solid human tumours. $\mathrm{Br} \mathrm{J}$ Cancer 95: 1611-25

28. Pepper MS, Skobe M (2003) Lymphatic endothelium: morphological, molecular and functional properties. J Cell Biol 163: 209-13

29. Makinen T, Adams RH, Bailey J, et al (2005) PDZ interaction site in ephrinB2 is required for the remodeling of lymphatic vasculature. Genes Dev 19: 397-410

30. Baldwin ME, Stacker SA, Achen MG (2002) Molecular control of lymphangiogenesis. Bioessays 24: 1030-40

31. Adams RH, Alitalo K (2007) Molecular regulation of angiogenesis and lymphangiogenesis. Nature Rev Mol Cell Biol 8: 464-78

32. Tammela T, Saaristo A, Holopainen T, et al (2007) Therapeutic differentiation and maturation of lymphatic vessels after lymph node dissection and transplantation. Nature Med 13: 1458-66

33. Karnezis T, Shayan R, Caesar C, et al (2012) VEGF-D promotes tumor metastasis by regulating prostaglandins produced by the collecting lymphatic endothelium. Cancer Cell 21: 181-95

34. Azzali G (2007) Tumor cell transendothelial passage in the absorbing lymphatic vessel of transgenic adenocarcinoma mouse prostate. Am J Pathol 170: 334-46

35. He Y, Rajantie I, Pajusola K, et al (2005) Vascular endothelial cell growth factor receptor 3-mediated activation of lymphatic endothelium is crucial for tumor cell entry and spread via lymphatic vessels. Cancer Res 65: 4739-46 
36. Hoshida $\mathrm{T}$, Isaka $\mathrm{N}$, Hagendoorn $\mathrm{J}$, et al (2006) Imaging steps of lymphatic metastasis reveals that vascular endothelial growth factor-C increases metastasis by increasing delivery of cancer cells to lymph nodes: therapeutic implications. Cancer Res 66: 8065-75

37. Shayan R, Karnezis T, Tsantikos E, et al (2007) A system for quantifying the patterning of the lymphatic vasculature. Growth Factors 25: 417-25

38. Achen MG, Jeltsch M, Kukk E, et al (1998) Vascular endothelial growth factor D (VEGF-D) is a ligand for the tyrosine kinases VEGF receptor 2 (Flk1) and VEGF receptor 3 (Flt4). Proc Natl Acad Sci USA 95: 548-53

39. Stacker SA, Stenvers K, Caesar C, et al (1999) Biosynthesis of vascular endothelial growth factor-D involves proteolytic processing which generates noncovalent homodimers. J Biol Chem 274: 32127-36

40. Saaristo A, Tammela T, Farkkila A, et al (2006) Vascular endothelial growth factor-C accelerates diabetic wound healing. American Journal of Pathology 169: 1080-7 41. Muthuchamy M, Gashev A, Boswell N, et al (2003) Molecular and functional analyses of the contractile apparatus in lymphatic muscle. Faseb J 17: 920-2

42. Karpanen T, Egeblad M, Karkkainen MJ, et al (2001) Vascular endothelial growth factor $\mathrm{C}$ promotes tumor lymphangiogenesis and intralymphatic tumor growth. Cancer Res 61: 1786-90

43. Wirzenius M, Tammela T, Uutela M, et al (2007) Distinct vascular endothelial growth factor signals for lymphatic vessel enlargement and sprouting. J Exp Med 204: $1431-40$ 
44. Farnsworth RH, Karnezis T, Shayan R, et al (2011) A role for bone morphogenic protein-4 in vascular endothelial growth factor-D mediated tumor growth, metastasis and vessel remodelling. Cancer Res 71: 6547-57

45. Achen MG, Stacker, S.A. (2012) Vascular endothelial growth factor-D:signalling mechanisms, biology and clinical relevance. Growth Factors (in press)

46. Shayan R, Rozen W, Bernard S, et al (2008) Perforator Dilatation Induced by Body Weight Gain Is Not Reversed by Subsequent Weight Loss: Implications for Perforator Flaps. Plast Reconstr Surg 122: 1765-72

47. Stadelmann WK, Reintgen DS (1998) Prognosis in malignant melanoma. Hematology/oncology clinics of North America 12: 767-96, vi

48. Deutsch A, Lubach D, Nissen S, et al (1992) Ultrastructural studies on the invasion of melanomas in initial lymphatics of human skin. J Invest Dermatol 98: 64-7

49. Gordon EJ, Rao S, Pollard JW, et al (2010) Macrophages define dermal lymphatic vessel calibre during development by regulating lymphatic endothelial cell proliferation. Development 137: 3899-910

50. Harvey NL, Gordon EJ (2012) Deciphering the roles of macrophages in developmental and inflammation stimulated lymphangiogenesis. Vasc Cell 4: 15 


\section{FIGURE LEGENDS}

\section{Figure 1. Skin-adherent VEGF-D ${ }^{+}$tumors generate higher densities of abnormal lymphatics, metastasising at higher rates than equivalent body-wall tumors}

(A) Macroscopic and histological appearance of 293-VEGF-D ${ }^{+}$tumors in mice. Tumors were adherent to either the skin undersurface ('SK' tumors), as seen in upper left and lower left panels; or body wall ('BW' tumors), as in upper right and lower right panels. (B) The relative frequency of metastasis from VEGF-D ${ }^{+}$SK, VEGF-D ${ }^{+}$BW and VEGF$\mathrm{D}^{-}$flank tumors, to axillary lymph nodes is shown graphically as the $\%$ of lymph node metastases $($ Upper panel). $* *$ denotes $\mathrm{p}$ value $<0.01$ Student's $\mathrm{t}$ test, Error bar $=$ SEM. Western blotting of tumor lysate for VEGF-D ( $\sim 55 \mathrm{kDa}$ band indicates full-length form of VEGF-D) indicates high levels of VEGF-D protein expression in both BW and SK tumors (lower left panel), and this is confirmed by ELISA analysis of serum from mice bearing BW or SK tumors, for tumor-derived human VEGF-D (Lower right panel). ** denotes $\mathrm{p}$ value $<0.01$ Student's $\mathrm{t}$ test, Error bar $=$ SEM. VEGFD ${ }^{-}$tumors are pooled samples from BW and SK tumor-bearing mice in lower right panel of Fig. 1B. (C) Quantification analysis of LYVE- $1^{+}$lymphatic vessels in VEGF-D ${ }^{+}$SK, VEGF-D ${ }^{+}$BW

and VEGF-D ${ }^{-}$flank tumors. Units shown in $10^{6}$ pixels/x10 objective field. ** denotes $\mathrm{p}$ value $<0.01$ Student's t test, Error bar = SEM. (D). LYVE-1, podoplanin and LVYE1/SMA immunohistochemical staining of VEGF-D ${ }^{+}$SK tumors and VEGF-D ${ }^{+}$BW tumors. Podoplanin immunostaining of lymphatics in SK tumors reveals lymphatics forming branched septa that permeated the tumor from the periphery, spanning much of the tissue section and forming LEC-lined spaces. Fluorescent co-staining for SMA (red) with LYVE-1 (green) in the lower right panel showed intermittent and variable SMCs 
associated with tumor lymphatics. Black arrows in upper right panel and lower left indicate tumor lymphatics and white arrows indicate LEC-lined spaces. SK, skin tumor; BW, Body Wall tumor. Scale Bars, $100 \mu \mathrm{m}$.

\section{Figure 2. Analysis of lymphatic subtypes in mouse and human tumors and tissues}

(A) Immunohistochemical staining identifies abundant initial lymphatics in mouse skin stained with either LYVE-1 (at low power (x4) in upper left panel, and high power (x20) in upper middle panel) or Podoplanin (upper right panel) in serial sections of mouse skin. LYVE-1 and podoplanin staining of body wall serial sections demonstrates LYVE-1/podoplanin ${ }^{+}$collecting lymphatic vessels in mouse flank tissue. Black arrows indicate tumor lymphatics; S, skin; B, body wall; A, artery; V, vein; podo, podoplanin. Quantification of the density of different lymphatic vessel subtypes present in different anatomical locations of the mouse skin. Units shown in LVD/10 x field. ** denotes $\mathrm{p}$ value $<0.01$ Student's t test, Error bar $=$ SEM. I, initial lymphatics; PC, pre-collector lymphatics; Coll, collecting lymphatic vessel. (C). Human breast 'skin to body wall' tissue was taken and represented an area from the skin $(\mathrm{S})$ to the fascia $(\mathrm{F})$. Tissue sections were stained with an antibody to LYVE-1. Scale Bar, $100 \mu \mathrm{m}$. (D) Quantification of human lymphatic subtypes immunostained with antibody against LYVE-1 and D2-40 demonstrated the density of small and collecting lymphatic subtypes in the superficial and deep human tissues; i.e. comparing the skin (in the dermis) with the body wall (deep to the deep fascia). Units shown in LVD/10 x field. I, initial lymphatics; PC, pre-collector lymphatics; Coll, collecting lymphatic vessel. LVD, lymphatic vessel density. $* *$ denotes $\mathrm{p}<0.01$ Student's $\mathrm{t}$ test, Error bar $=$ SEM. 
Figure 3. Analysis of lymphatics in mouse ear tumors and wounds, and human

\section{melanomas}

(A) Macroscopic appearance (left panel) and LYVE-1 immunostained section (right panel) of VEGF-D ${ }^{+} 293$-EBNA tumors grown in the mouse ear. Open arrows indicate dilated dermal initial lymphatics and arrowheads denote pathological neo-lymphatics formed at margin of the tumor. Inset in left panel depicts enlarged view of ear tumor denoted by broken line box. T, tumor. Scale Bar, $50 \mu \mathrm{m}$. (B) Whole-mount immunofluorescent staining of VEGF-D ${ }^{+}$mouse ear tumors for LYVE-1 (green) in upper panels; arrows indicate 'sheet-like' lymphatic morphology and arrowheads denote the normal native lymphatic network at the tumor margin. Inset in upper panel demonstrates lymphatic endothelial structures (denoted by white arrows), at higher power. Lower left panel depicts sprouting from normal initial lymphatics immediately adjacent to the VEGF-D ${ }^{+}$tumor. Wholemount immunofluorescent staining of lymphatics in the skin near the VEGF-D ${ }^{+}$mouse ear tumor, for LYVE-1 (green); open arrows indicate lymphatic vessels sprouting towards the tumor. Inset in left panel depicts enlarged view of ear tumor denoted by white box. Broken line denotes tumor margin. T, tumor. Right lower panel depicts normal collecting lymphatic vessels stained for podoplanin (green) (demarcated by white arrows), immediately adjacent to the margin of the VEGF-D ${ }^{+}$mouse ear tumor, undergoing no discernable sprouting. Scale Bars, $50 \mu \mathrm{m}$. (C) Quantitative analysis of lymphatic sprouts arising from LYVE- $1^{+}$small lymphatic vessels adjacent to VEGF-D ${ }^{+}$ear tumors and ear punch biopsy wounds. $*^{*}=\mathrm{p}<0.01$; $^{*}=\mathrm{p}<0.05$ comparing lymphatic sprouting parameters between the tumor and wound models in the ear (Student's $\mathrm{t}$ test); T, tumor; W, wound; S, small lymphatics; C, collecting lymphatics. (D) Left panel depicts wholemount LYVE-1 immunofluorescent staining of lymphatics adjacent to a punch wound 
in a mouse ear; open white arrows indicate lymphatic sprouts, closed white arrows indicate normal lymphatics from which lymphatic sprouts arose at the points denoted by arrowheads. Right panel depicts immunohistochemical staining of metastatic human melanoma for podoplanin. Scale Bar, $100 \mu \mathrm{m}$ in left panel and $50 \mu \mathrm{m}$ in right panel.

\section{Figure 4.}

\section{Schematic representation of lymphatic vessel subtypes found in dermal and subcutaneous layers of normal mammalian skin.}

(A) Superficial dermal initial lymphatics absorb lymph for transportation to the pre-collecting vessels. The pre-collecting vessels are deeper in the dermis and drain into subcutaneous collecting lymphatics, which have valves, a thicker wall including SMC coverage and a continuous basement membrane. The SK tumor is seen near the skin, and generates 'neolymphatic vessels' from the small calibre dermal lymphatics under the influence of VEGF-D. The BW tumor arises deeply, and whilst it may lie adjacent to 'non-sprouting' collecting lymphatics, there are no lymphatics nearby with the capacity to sprout and infiltrate the tumor. The BW tumor is therefore unable to access the lymphatic network and spread to regional lymph nodes. IL, initial lymphatic; PC, pre-collector lymphatic. (B) Summary of lymphatic vessels (LV) occurring in the vicinity of SK and BW tumors. 


\section{Supplemental Material}

\section{Figures}

1) Fluorescent co-staining of metastatic VEGF-D ${ }^{+}$SK tumor section for podoplanin (green) and LYVE-1 (red) indicates co-localization on tumor lymphatics. Nuclei are stained using DAPI (Blue). Bar, $100 \mu \mathrm{m}$.

2) Fluorescent co-staining of VEGF-D ${ }^{+}$SK tumor for SMA (red) and podoplanin (green) showed intermittent and variable SMC associated with tumor lymphatics. Nuclei are stained using DAPI (Blue). Bar, $100 \mu \mathrm{m}$.

3) D2-40 antibody staining of podoplanin on lymphatics (arrows) in normal human breast skin samples. Bar, $100 \mu \mathrm{m}$.

4) D2-40 staining of podoplanin on collecting lymphatics in normal human breast samples. Bar, $100 \mu \mathrm{m}$.

5) Podoplanin staining of tumor lymphatics in section of VEGF- ${ }^{+}$mouse ear tumor. Unfilled arrow indicates LEC-lined space. Bar, $200 \mu \mathrm{m}$. 